\title{
THE STIELTJES TRANSFORM OF DISTRIBUTIONS
}

\author{
U. N. TIWARI \\ Department of Mathematics \\ John Abbott College \\ Montreal, Canada \\ J. N. PANDEY \\ Department of Mathematics \\ Carleton University \\ Ottawa, Canada
}

(Received September 28, 1978 and in revised form April 3, 1979)

ABSTRACT. In the present work, two complex inversion formulas of Byrne and Love for generalized Stieltjes transformation are shown to be valid for a class of distributions. This is accomplished by transfering the complex inversic formulas on the testing function space of a class of distributions and then showing that the limiting process in the resulting formula converges in the topology of the testing function space.

KEY WORDS AND PHRASES. Generalized Functions, Stieltjes Transformation. AMS (MOS) SUBJECT CLASSIFICATION (1970) CODES. Primary 46A40, Secondary $42 A 25$.

1. INTRODUCTION.

Let $\mathrm{p}$ be any complex number except zero and the negative integers. Then 
for all $s$ in the "cut plane", that is all complex numbers except those which are negative real or zero, the Stieltjes Transform in its general form is defined by:

$$
F(0)=\int_{0(0+t)^{p}}^{\infty} \frac{f(t) d t}{(x)}
$$

The following inversion theorems for particular values of $p$ and $s$ are well known. THEOREM A (Widder). If $f(t)$ belongs to $L(0, R)$ for every positive $R$ and is such that the integral

$$
F(x)=\int_{0}^{\infty} \frac{f(t) d t}{t+x} d t
$$

converges for $x>0$, then $F(s)$ exists for conplex $s$ in the cut plane and

$$
\lim _{n \rightarrow 0+} \frac{f(\xi-1 \eta)-F(-\xi+1 \eta)}{2 \pi^{1}}=\frac{f(\xi+)+f(\xi-)}{2}
$$

for any positive $\xi$ at which $f\left(\xi^{+}\right)$and $f\left(\xi^{-}\right)$both exist.

THEOREM B (Sumner). If $p>0, f(t)$ is locally integrable in $[0, \infty]$, the improper Lebesgue integral.

$$
F(s)=\int_{0}^{\infty} \frac{f(t) d t}{(s+t)^{p}}
$$

converges (for a certain value of $s$ in the cut $p l a n e$ and so for $a 11$ ), $t>0$ and the limits $f(t+0)$ exist, then:

$$
\frac{1}{2}[f(t+0)+f(t-0)]=\lim _{\eta \rightarrow 0+} \frac{-1}{2 \pi 1} \int_{0}^{t} d x \int_{\eta x}(x+z)^{p-1} F^{\prime}(z) d z
$$


where $C_{\eta x}$ is a contour in the cut plane from $-x-i \eta$ to $-x+i \eta$.

THEOREM 1.3 (Byrne and Love). If Re $p>1$, f is locally integrable in $[0, \infty]$, improper Lebesgue integral (1.1) converges, and $\lambda>0$; then, for each positive $x$ for which the Lebesgue limits $f(x+0)$ exists,

$$
\frac{1}{2}\{f(x+0)+f(x-0)\}=\lim _{\eta \rightarrow 0+} \frac{p-1}{2 \pi i} \int_{-x}^{\lambda}(x+t)^{p-2}(F(t-1 \eta)-F(t+1 \eta)\} d t . \quad[2, p .349]
$$

THEOREM 1.4 (Byrne and Love). If Re $p>1, \frac{f(t)}{1+t^{2}} \in L(0, \infty)$ and the Improper Lebesgue integral

$$
F(s)=\int_{0}^{\infty} \frac{f(t)}{(s+t)^{p}} d t
$$

converges, then for each positive $x$ for which the Lebesgue limit $f(x+0)$ exists,

$$
\frac{1}{2}\{f(x+0)+f(x-0)\}=\lim _{\eta \rightarrow 0+} \int_{-x}^{\infty}(x+t)^{p-2}(F(t-i \eta)-F(t+i \eta)\} d t \quad[2, p \cdot 352]
$$

THEOREM 1.1 has been extended to distributions by Pandey and Zemanian [13] and Pandey [14]. Theorem 1.2 was extended to distribution by Pathak [15]. Our object is to extend theorems 1.3 and 1.4 of Byrne and Love to generalized functions (distributions).

\section{THE TESTING FUNCTION SPACE, $S_{\alpha}$ (I) AND ITS DUAL.}

An infinitely differentiable complex valued function $\Phi(x)$ defined over $I=(0, \infty)$ belongs to the testing function spaces $S_{\alpha}(I)$ if,

$$
\gamma_{k}(\Phi)=\sup _{0<x<\infty}\left|(1+x)^{\alpha} x^{k}\left(\frac{d}{d x}\right)^{k} \Phi(x)\right|<\infty
$$


for all $k=0,1,2, \ldots$, where $\alpha$ is a fixed real number. Clearly, $s_{\alpha}(I)$ is a vector space with respect to the field of complex numbers. The zero element of the vector space $S_{\alpha}(I)$ is the function defined over I which is identically zero. The topology over $S_{\alpha}(I)$ is generated by the collection of seminorms $\left.\mid \gamma_{k}\right\}_{k=0}^{\infty}$ [24; p. 8]. We say that a sequence $\left\{\Phi_{\nu} \mid\right.$ where $\Phi_{\nu}$ belongs to $s_{\alpha}$ (I) converges in $S_{\alpha}(I)$ to $\Phi(x)$ if for each fixed $k, \gamma_{k}(\Phi,-\Phi)$ tends to zero as $\nu$ tends to $\infty$. The space $S_{\alpha}(I)$ is a locally convex Hausdorff topological vector space. The space $D(I)$ is a vector subspace of $S_{\alpha}(I)$ and the topology of $D(I)$ is stronger than the topology induced on $D(I)$ by $S_{\alpha}(I)$ and as such the restriction of any member of $S_{\alpha}^{\prime}(I)$ to $D(I)$ is in $D^{\prime}(I)$, where $S \alpha(I)$ and $D^{\prime}(I)$ denote the dual spaces of $S_{\alpha}(I)$ and $D(I)$ respectively. We say that a sequence $I_{\nu} \rho_{\nu=I}^{\infty}$ where $\Phi_{\nu}(x)$ belongs to $S_{\alpha}(I)$ is a Cauchy sequence in $S \alpha(I)$ if $\gamma_{k}\left(\Phi_{\nu}-\Phi_{\nu}\right)$ goes to zero for any non-negative integer $k$ as $\mu$ and $\nu$ both tend to infinity independently of each other. It can be readily seen that $S_{\alpha}(I)$ is sequentially complete.

\section{THE DISTRIBUTIONAL STIELTJES TRANSFORMATION}

For a complex s not negative or zero, $\frac{1}{(s+x)^{p}}$ belongs to $s_{\alpha}^{\prime}$ where a < Rep. Therefore, the distributional stieltjes transformation $F(s)$ of an arbitrary element $f \in S_{\alpha}^{\prime}, a<\operatorname{Re} p$, is defined by

$$
F(s) \triangleq<f(x), \frac{1}{(s+x)^{p}}>
$$

where $s$ belongs to the complex plane cut along the negative real axis including the origin.

THEOREM 3.1. If $\mathrm{m}$ and $\mathrm{k}$ both assume non-negative integral values and $\Omega$ is a 
compact set of the complex plane not meeting the negative real axis, then for fixed non-negative integers $m$ and $k$, there exists a constant ${ }_{\Omega}$ satisfying

$$
\gamma_{\mathrm{m}}\left[\frac{1}{(s+\mathrm{x})^{\mathrm{p}+\mathrm{k}}}\right] \leq \mathrm{B}_{\Omega}<\infty
$$

uniformly for all s lying in the compact set $\Omega$ of the complex plane not meeting the negative real axis on the origin.

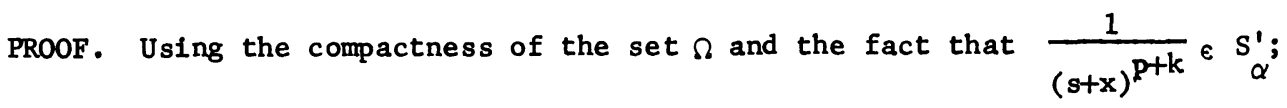
$\alpha<\operatorname{Re} \mathrm{p}$, the theorem is immediate.

THEOREM 3.2. For an arbitrary $f \in S_{\alpha}^{\prime}$ and $a<\operatorname{Re} p$ let $F(s)$ be defined by the equation (3.1). Then, for $m=1,2, \ldots$

$$
\left(\frac{d}{d s}\right)^{m} F(s)=\left\langle f(x), \frac{(-1)^{m}(p)_{m}}{(s+x)^{p+m}}\right\rangle
$$

where $(p)_{m}=p(p+1)(p+2) \ldots(p+m-1)$.

PROOF. If $p$ is such that $s^{p}$ does not have a branch cut in the complex plane, the proof can be given in a way similar to that given in [3, Lemma 2a]. If $p$ is such that $s^{p}$ has a branch cut (along the negative real axis for the sake of definiteness), then choose the contour of integration as shown in the complexplane cut along the negative real axis below. 


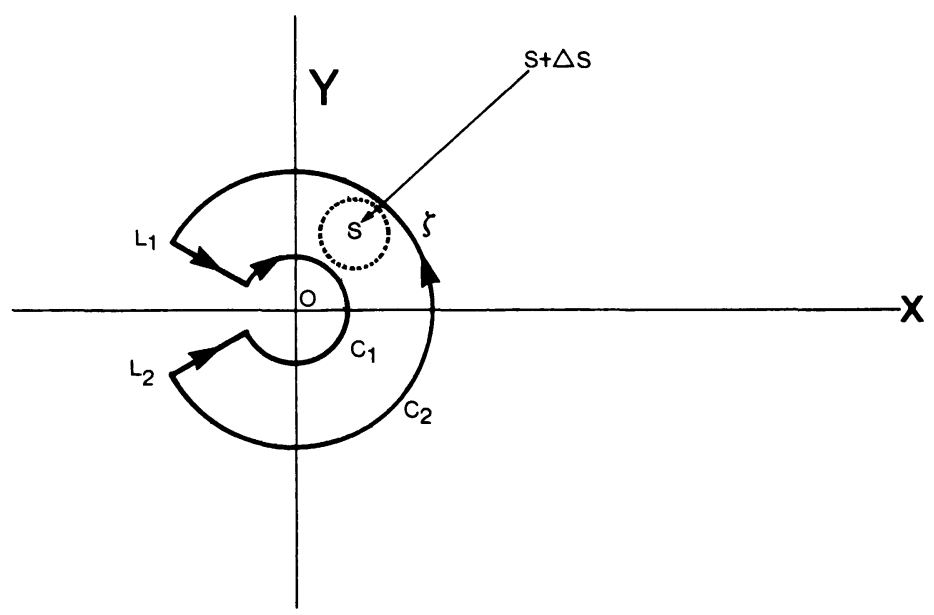

Here, $C_{1}$ and $C_{2}$ are arcs of two concentric circles with centre at origin and $C$ is the contour of integration as shown. The radil of $C_{1}$ and $C_{2}$ and paths $L_{1}$ and $L_{2}$ are so chosen that the point $s$ is contained in the region bounded by the contour. Let $\mathrm{d}=\operatorname{linf}_{\xi_{\epsilon} \mathrm{C}}|\xi-s|$ and choose $|\Delta s|<\frac{\mathrm{d}}{2}$. Now

$$
\begin{aligned}
& \left.\frac{F(s+\Delta s)-F(s)}{\Delta s}-<f(t), \frac{-p}{(s+t)^{p+1}}\right\rangle \\
& =\left\langle f(t), \frac{1}{\Delta s} \cdot \frac{1}{2 \pi^{i}} \cdot \int_{C} \frac{1}{(z+t)^{p}}\left[\frac{1}{z-s-\Delta s}-\frac{1}{z-s}-\frac{\Delta s}{(z-s)^{2}}\right] d z>\right.
\end{aligned}
$$

where $C$ is the contour shown in the diagram

$$
=\left\langle f(t), \theta_{\Delta s}\right\rangle
$$

where

$$
\theta_{\Delta s}=\frac{\Delta s}{2 \pi i} \int_{C} \frac{1}{(z+t)^{p}} \frac{1}{(z-s)^{p}(z-s-\Delta s)} d z
$$

We now wish to show that $\theta_{\Delta s} \rightarrow 0$ in $S_{\alpha}(I)$ as $\Delta s \rightarrow 0$.

Using Theorem 3.1, we have

$$
\gamma_{k}\left(\theta_{\Delta s}\right) \leq B_{c} \frac{|\Delta s|}{2 \pi} \frac{L}{d^{3}},
$$


where $L$ is the length of the contour $C$ and $B_{C}$ is the uniform bound of

$$
\frac{(p)_{k}(1+t)^{\alpha_{t}^{k}}}{(z+t)^{p+k}} \quad \text { for all z lying on the closed contour } c \text { and } t>0 \text {. }
$$

Letting $\Delta s \rightarrow 0$ in (3.3) and using (3.4), we get

$$
F^{\prime}(s)=\left\langle f(t), \frac{-p}{(g+t)^{p+1}}\right\rangle
$$

Now; the theorem follows from induction on the order of the derivative of $F(s)$.

THEOREM 3.3. The function $F^{(m)}(x)$ for real $x$ where $F(s)$ is the stieltjes transform of $f \in S_{\alpha}^{\prime}$, satisfies the following relation:

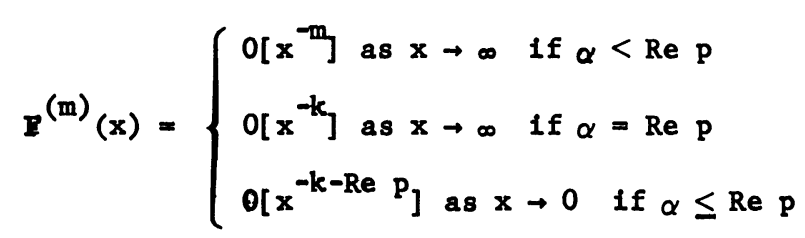

The proof is immediate from the boundedness roperty of distributions [9, p. 18].

\section{COMPLEX INVERSION THEOREYS}

We are now ready to prove our first inversion theorem.

THEOREM 4.1. For a fixed $\alpha<1$ and Re $p>1$, let $f \in S_{\alpha}^{\prime}(I)$ and let $F(s)$ be the Stieltjes transform of $f(t)$ as defined by (3.1). Then,

$$
\begin{aligned}
\lim _{\eta \rightarrow 0}<\frac{p-1}{2 \pi^{I}} \int_{-x}^{\infty}(x+t)^{p-2} & {[F(t-1 \eta)-F(t+1 \eta)] d t, \Phi(x)>} \\
& =\langle f, \Phi>\text { iur: } 11 \Phi \in D(I)
\end{aligned}
$$

ProOF. First consider

$$
\int_{-x}^{\lambda}(x+t)^{p-2} F(t-i \eta) d t=\int_{-x}^{\lambda}(x+t)^{p-2}<f(y), \frac{1}{(y+t-1 \eta)^{p}}>d t
$$


For fixed $x$ and $t$,

$$
\frac{(x+t)^{p-2}}{(y+t-i \eta)^{p}} \in s_{\alpha}^{(I)}
$$

Since, in view of Theorem $3.2, F(s)$ is analytic in the cut plane, the left-hand side integral in (4.1) is meaningful. By using the technique of Riemann sums it can be shown that for $\epsilon>0$,

$$
\begin{aligned}
& \int_{-x+\epsilon}^{\lambda}(x+t)^{p-2} F(t-i \eta) d t \\
& =\left\langle f(y), \int_{-x+\epsilon}^{\lambda} \frac{(x+t)^{p-2}}{(y+t-i \eta)^{p}} d t>\right. \\
& =\left\langle f(y), \frac{1}{p-1} \cdot \frac{1}{(x-y+i \eta)}\left[\frac{(x+\lambda)^{p-1}}{(y+\lambda-i \eta)^{p-1}}-\frac{e^{p-1}}{(y-x+\varepsilon-i \eta)^{p-1}}\right]>\right. \\
& =I \text { (say). }
\end{aligned}
$$

One can easily check that as $\epsilon \rightarrow 0+, \frac{e^{p-1}}{(y-x+\epsilon-1 \eta)^{p-1}} \rightarrow 0$ in $s_{\alpha}(I)$ for $\operatorname{Re} p>1>\alpha$ and for fixed $\lambda, \eta$ and $x$. Therefore, letting $\varepsilon \rightarrow 0$, we get:

$$
\int_{-x}^{\lambda}(x+t)^{p-2} F(t-i \eta) d t=\left\langle f(y), \frac{(\lambda+x)^{p-1}}{(p-1)(y-x-i \eta)(y+\lambda-i \eta)^{p-1}}\right\rangle
$$

In view of Lemma $3.5 *[7$, p. 12], it follows that: as $\lambda \rightarrow \infty$

$$
\frac{1}{(y-x-i \eta)}\left(\frac{\lambda+x}{(y+\lambda-i \eta)}\right)^{p-1} \rightarrow \frac{1}{y-x-i \eta} \text { in } s_{\alpha}(I)
$$

for fixed $x$ and $\eta$.

Therefore, letting $\lambda \rightarrow \infty$ in (4.2), we obtain

$$
\int_{-x}^{\infty}(x+t)^{p-2} F(t-1 \eta) d t=\left\langle f(y), \frac{1}{p-1} \cdot \frac{1}{y-x-1 \eta}\right\rangle
$$

* The proof was provided by Professor E.R. Love. 
Using a similar argument, we can show that

$$
\int_{-x}^{\infty}(x+t)^{p-2} F(t+i \eta) d t=\left\langle f(y), \frac{1}{p-1} \cdot \frac{1}{y-x+1 \eta}>\right.
$$

Combining Equations (4.4) and (4.5), we get

$$
\begin{aligned}
& \frac{p-1}{2 \pi^{i}} \int_{-x}^{\infty}(x+t)^{p-2}[F(t-i \eta)-F(t+i \eta)] d t \\
& =\left\langle f(y), \frac{\eta}{\pi\left[(y-x)^{2}+\eta^{2}\right]}>\right.
\end{aligned}
$$

Now using the technique of Riemann sums, we obtain

$$
\begin{aligned}
& <\frac{\mathrm{p}-1}{2 \pi i} \int_{-x}^{\infty}(x+t)^{\mathrm{p}-2}[F(t-i \eta)-F(t+i \eta)] d t, \Phi(x)> \\
& =\left\langle f(y), \int_{a}^{b} \frac{\eta \Phi(x) d x}{\pi\left[(y-x)^{2}+\eta^{2}\right]}\right\rangle
\end{aligned}
$$

where the support of $\Phi(x) \in D(I)$ is contained in $(a, b), b>a>0$. Using the same techniques as followed in proving Theorem 2 of (3) one can show that

$$
\frac{1}{\pi} \int_{a}^{b} \frac{\eta \Phi(x) d x}{(y-x)^{2}+\eta^{2}} \rightarrow \Phi(y)
$$

in the topology of $S_{\alpha}(I)$ as $\eta \rightarrow 0+$. Therefore, letting $\eta \rightarrow 0+$ in (4.7), we have

$$
\left.\lim _{\eta \rightarrow 0+}<\frac{\mathrm{p}-1}{2 \pi^{i}} \int_{-x}^{\infty}(x+t)^{p-2}[F(t-i \eta)-F(t+i \eta)] d t, \Phi(x)\right\rangle=\langle f, \Phi\rangle
$$

This completes the proof of the theorem.

To prove our other inversion theorems, we require a couple of Lemmas. LEMMA 4.2. Let $t, s, \eta>0$. Then, for finite $b>a>0$ and $\alpha<1$, $\lim _{\eta \rightarrow 0+}(1+x)^{\alpha} \int_{a}^{b} \frac{|t-x|}{(t-x)^{2}+\eta^{2}} d t=0$ uniformly for all $x>0$. 
PROOF. Let

$I=(1+x)^{\alpha} \eta \int_{a}^{b} \frac{|t-x| d t}{(t-x)^{2}+\eta^{2}}$

Since $\sup _{x \gg N>b}\left|(1+x)^{\alpha} \frac{(t-x)}{(t-x)^{2}+\eta^{2}}\right|$ is bounded, for $\epsilon>0$, there exists a $a \leq t \leq b$

positive $N>b$ and $0<q<1$ such that

$|I|<\epsilon$

uniformly for all $\eta \in(0, q)$ and $x>N$.

Now assume that $\delta$ is a positive number $\leq \min \left(1, \frac{a}{2}\right)$ and for $\delta \leq x \leq N$ let us write.

$I=(1+x)^{\alpha} \eta\left(\int_{a}^{a+x-\delta}+\int_{a+x-\delta}^{a+x+\delta}+\int_{a+x+\delta}^{b}\right) \frac{|t-x|}{(t-x)^{2}+\eta^{2}} d t$.

Denote the three expressions on the right-hand side of Eqn. $(4.10)$ by $I_{1}, I_{2}$ and $I_{3}$ respectively.

Now

$$
\begin{aligned}
& I_{2}=\eta \int_{a+x-\delta}^{a+x+\delta} \frac{|t-x|(1+x)^{\alpha}}{(t-x)^{2}+\eta^{2}} d t \\
& \leq \eta 2 \delta \frac{1}{2 \eta}(1+N)^{\alpha}=\delta(1+N)^{\alpha}
\end{aligned}
$$

Now choose $\delta$ such that $\delta(1+N)^{\alpha}<\epsilon$ and fix $\delta$ this way. Therefore

$$
\left|I_{2}\right|<\epsilon
$$

uniformly for all $x \in[\delta, N]$ and $\eta \in[0, q]$.

$$
I_{3}=\eta \int_{a+x+\delta}^{b} \frac{(1+x)^{\alpha}|t-x|}{(t-x)^{2}+\eta^{2}} d t=(1+x)^{\alpha} \eta\left[\int_{a+x+\delta}^{x}+\int_{x}^{b}\right] \frac{|t-x|}{(t-x)^{2}+\eta^{2}} d t
$$


Therefore,

$$
I_{3}=\left\{\begin{array}{l}
=\frac{n}{2} \ln \left(\frac{(b-x)^{2}+\eta^{2}}{(a+\delta)^{2}+\eta^{2}}\right)(1+x)^{\alpha}, \text { if } \delta<x<b \\
=(1+x)^{\alpha} \frac{\eta}{2} \ln \left(\frac{\eta^{4}}{\left[(a+\delta)^{2}+\eta^{2}\right]\left[(b-x)^{2}+\eta^{2}\right]}\right) \text { if } x \geq b
\end{array}\right.
$$

Therefore, $I_{3} \rightarrow 0$ as $\eta \rightarrow 0+$

uniformly for all $x \in[\delta, N]$.

Next,

$$
I_{1}=(1+x)^{\alpha} \eta \int_{a}^{a+x-\delta} \frac{|t-x| d t}{(t-x)^{2}+\eta^{2}}
$$

Now for $\delta \leq x \leq a$

$$
I_{1}=(1+x)^{\alpha} \frac{\eta}{2} \ln \left(\frac{(a-\delta)^{2}+\eta^{2}}{(a-x)^{2}+\eta^{2}}\right) \rightarrow 0 \text { as } \eta \rightarrow 0+
$$

uniformly for all $x$ lying in $[\delta, a]$.

For $a \leq x \leq N$,

$$
\begin{aligned}
& I_{1}=\left[-\frac{\eta}{2} \ln \left(\frac{\eta^{2}}{(a-x)^{2}+\eta^{2}}\right)+\frac{n}{2} \ln \left(\frac{(a-\delta)^{2}+\eta^{2}}{\eta^{2}}\right)\right](1+x)^{\alpha} \\
& \rightarrow 0 \text { as } \eta \rightarrow 0+\text { uniformly for all } x_{\in} \in[a, N]
\end{aligned}
$$

Combining results (4.11) through (4.14) we have

$$
\overline{1 i m}_{n \rightarrow 0+}|I| \leq \varepsilon \quad \text { uniformly for all } x \geq 0
$$

Now, for $0<x \leq \delta$, we can see that

$$
|I| \leq(1+\delta)^{\alpha} \eta \text { \&n }\left[\frac{(b-x)^{2}+\eta^{2}}{(a-x)^{2}+\eta^{2}}\right] \rightarrow 0
$$

As $\eta \rightarrow 0+$ uniformly for all $x \in[0, \delta]$. 
Combining results (4.15) and (4.16), we get

$$
\overline{1 i m}_{n \rightarrow 0_{+}}|I| \leq \epsilon \quad \text { uniformly for all } x>0 .
$$

Thus, the proof of the lemma is complete.

LEMMA 4.3. Let $\operatorname{Re} p>1>\alpha$. Assume that $t, x, \lambda$ and $\eta$ are all positive numbers and $\Phi(t) \in D(I)$. Then,

$$
\begin{gathered}
I(x, t) \triangleq \frac{1}{2 \pi I} \int_{a}^{b}\left\{\frac{1}{x-t+i \eta}\left[\left(\frac{t+\lambda}{x+\lambda+i \eta}\right)^{p-1}-1\right]\right. \\
\left.-\frac{1}{x-t-1 \eta}\left[\left(\frac{t+\lambda}{x+\lambda-i \eta}\right)^{p-1}-1\right]\right\} \Phi(t) d t \rightarrow 0
\end{gathered}
$$

as $\eta \rightarrow 0+$ in the topology of $S_{\alpha}$ where the support of $\Phi(t) \in D(I)$ is contained in $(a, b) ; \quad b>a>0$.

PROOF. We have to show that for each $m=0,1,2, \ldots(1+x) \alpha^{\alpha}{ }^{m}{ }_{x}^{m} I(x, \eta) \rightarrow 0$ as $\eta \rightarrow 0$ uniformly.

It can be easily shown that

$$
D_{x}^{m} I(x, \eta)=0\left(\frac{1}{x^{m+1}}\right) \text { as } x \rightarrow \infty
$$

uniformly for all $\eta$ satisfying $0<\eta<1$. So,

$$
(1+x)^{\alpha}{ }_{x}^{m} D_{x}^{m} I(x, \eta) \rightarrow 0 \quad \text { as } x \rightarrow \infty
$$

uniformly for all $\eta \in(0,1)$. Therefore, for $\epsilon>0$ there exists $N>0$ such that

$$
\left|(1+x) \alpha_{x}^{m}{ }_{x}^{m} I(x, \eta)\right|<\epsilon
$$

uniformly for all $x \geq N$, and $0<\eta<1$.

Now consider the case $0<x \leq \eta$. We will first give the proof for $m=0$ and complete the proof for $m=1,2,3, \ldots$ by using the result for the case $\mathrm{m}=0$. 
I. For $m=0$. Write

$$
\begin{aligned}
(1+x)^{\alpha} I(x, \eta) & =\frac{(1+x)^{\alpha}}{2 \pi^{1}} \int_{a}^{b} \frac{x-t}{(x-t)^{2}+\eta^{2}}\left[\left(\frac{t+\lambda}{x+\lambda+i \eta}\right)^{p-I}-\left(\frac{\lambda+t}{\lambda+x-i \eta}\right)^{p-1}\right] \Phi(t) d t \\
& -(1+x)^{\alpha} \frac{\eta}{2 \pi} \int_{a}^{b} \frac{\Phi(t)}{(x-t)^{2}+\eta^{2}}\left[\left(\frac{t+\lambda}{x+\lambda+i \eta}\right)^{p-1}-1+\left(\frac{t+\lambda}{x+\lambda-i \eta}\right)^{p-1}-1\right] \Phi(t) d t \\
& =I_{1}(x, \eta)-I_{2}(x, \eta)
\end{aligned}
$$

First, consider $I_{1}(x, \eta)$. since,

$$
\begin{aligned}
\left|\frac{1}{(x+\lambda+i \eta)^{p-1}}-\frac{1}{(x+\lambda-i \eta)^{p-1}}\right| & =\left|(p-1) \int_{x+\lambda-i \eta}^{x+\lambda+i \eta} z^{-p} d z\right| \\
& \leq 2|p-1| \eta \frac{e^{\pi \mid I m p} \mid}{(x+\lambda)^{\operatorname{Re} p}} \\
& \leq 2|p-1| \eta e^{\pi|\operatorname{Im} p|} / \lambda^{\operatorname{Re} p}
\end{aligned}
$$

Therefore we can find a constant $B(p)$ independent of $x$ and $\eta$ such that

$$
\left|I_{1}(x, \eta)\right|<B(1+x)^{\alpha} \eta \int_{a}^{b} \frac{|x-t| d t}{(x-t)^{2}+\eta}
$$

In view of Lemma 4.2, the right-hand side converges to 0 uniformly for all $\mathbf{x}>0$ as $\eta \rightarrow 0+$.

We now consider $I_{2}(x, \eta)$. For $0<\eta<b$, using Lemma $3.3(7, p$. 9) we get

(i) For $\operatorname{Re} \mathrm{p} \geq 2, \mathrm{t} \leq \mathrm{x}$

$$
\begin{aligned}
\left|\left(\frac{t+\lambda}{x+\lambda+i \eta}\right)^{p-1}-1\right| & \leq|p-1| e^{2 \pi|I m p|} \frac{|t-x|+\eta}{\sqrt{(x+\lambda)^{2}+\eta^{2}}}, t \leq x \\
& \leq|p-1| e^{2 \pi|I m p| \frac{|t-x|+\eta}{\lambda}},
\end{aligned}
$$


(i1) For $\operatorname{Re} \mathrm{p} \geq 2, \mathrm{t} \geq \mathrm{x}$

$$
\begin{aligned}
& \left|\left(\frac{t+\lambda}{(x+\lambda+1 \eta)}\right)^{p-1}-1\right| \leq|p-1| e^{2 \pi|\operatorname{Im} p|}\left|\frac{t+\lambda+1 \eta}{x+\lambda+1 \eta}\right|^{R e p-2} \cdot \frac{|t-x|+n}{\sqrt{(\lambda+x)^{2}+\eta^{2}}} \\
& \leq \frac{|\mathrm{p}-1| \mathrm{e}^{2 \pi|\operatorname{Im} \mathrm{p}|}}{\lambda^{\operatorname{Re} \mathrm{p}-1}}(\mathrm{~b}+\lambda+1)^{\operatorname{Re} \mathrm{p}-2}(|\mathrm{t}-\mathrm{x}|+\eta) ; 0<\eta<1
\end{aligned}
$$

(i1i) For $\operatorname{Re} p \leq 2, t \leq x$ and $t \in[a, b], b>a>0,0<\eta<1$.

$$
\left|\left(\frac{t+\lambda}{x+\lambda+1 \eta}\right)^{p-1}-1\right| \leq \frac{|p-1| e^{2 \pi|I m p|}(a+\lambda)^{\operatorname{Re} p-2}}{\lambda^{\operatorname{Re} p-1}\left[1+\frac{1}{2}\right]^{\operatorname{Re}(p-2)^{2}}} \cdot \frac{(|t-x|+n)}{\left(1+\frac{b}{\lambda}\right)^{\operatorname{Re} p-2}}
$$

(iv) For re $p \leq 2, t \geq x, t \in[a, b], b>a>0,0<\eta<1$

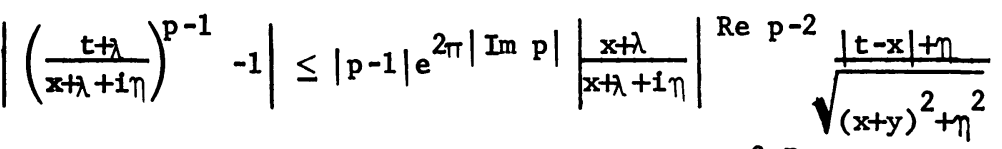

$$
\begin{aligned}
& \leq \frac{|\mathrm{p}-1| \mathrm{e}^{2 \pi|\operatorname{Im} \mathrm{p}|}}{\lambda}\left[1+\frac{1}{\lambda^{2}}\right]^{2-\operatorname{Re} \mathrm{p}}(|\mathrm{t}-\mathrm{x}|+\eta)
\end{aligned}
$$

Therefore, from inequalities (4.19) through (4.21), it is evident that for Re $p>1$ and $0<\eta<1$, there exists a positive constant $k(\lambda, p)$ independent of $t$ and $x$ satisfying

$$
\left|\left(\frac{t+\lambda}{x+\lambda+i \eta}\right)^{p-1}-1\right| \leq k(\lambda, p)[(t-x)+\eta] \quad t \in(a, b)
$$

Similarly, under the same set of conditions

$$
\left|\left(\frac{t+\lambda}{x+\lambda-1 \eta}\right)^{p-1}-1\right| \leq k(\lambda, p)[(t-x)+\eta], t \in(a, b)
$$

In view of these inequalities we, therefore, have established that 


$$
\left|I_{2}(x, \eta)\right| \leq \frac{K\left(\alpha_{2} p\right) M(1+x)^{\alpha}}{\pi}(1+x)^{\alpha} \int_{a}^{b} \frac{n(|t-x|+\eta) d t}{(t-x)^{2}+\eta^{2}}
$$

where $M=\sup |\Phi(t)|$

$$
a \leq t \leq b
$$

That is

$$
\left|I_{2}(x, \eta)\right| \leq \frac{x\left(\lambda_{2}, p\right) M}{\pi}(1+x)^{\alpha}\left\{\eta \int_{a}^{b} \frac{|t-x| d t}{(t-x)^{2}+\eta^{2}}+\eta^{2} \int_{a}^{b} \frac{d t}{(t-x)^{2}+\eta^{2}}\right\}
$$

since

$$
(I+x)^{\alpha} \eta^{2} \int_{a}^{b} \frac{d t}{(t-x)^{2}+\eta^{2}} \rightarrow 0
$$

as $\eta \rightarrow 0+$ uniformly for all $x>0$. Therefore, in view of Lemma 5.2 , it follows that

$$
I_{2}(x, \eta) \rightarrow 0 \text { as } \eta \rightarrow 0+\text { uniformly for all } x>0 .
$$

II) The case $\mathrm{m}=1,2,3, \ldots$

A careful computation along with integration by parts will show that

$$
\begin{aligned}
(1+x)^{\alpha}{ }_{x D} I(x, \eta) & =\frac{(1+x)^{\alpha}}{2 \pi^{1}} \times \int^{b}\left\{\frac{1}{x-t+1 \eta}\left[\left(\frac{t+\lambda}{x+\lambda+1 \eta}\right)^{p-1}-1\right]\right. \\
& \left.-\frac{1}{x-t-1 \eta}\left[\left(\frac{t+\lambda}{x+\lambda-1 \eta}\right)^{p-1}-1\right]\right\} \Phi^{\prime}(t) d t \\
& +\frac{(p-1)}{2 \pi 1}(1+x)^{\alpha} \times \int_{a}^{b}\left[(x+\lambda+1 \eta)^{-p}-(x+\lambda-1 \eta)^{-p}\right](t+\lambda)^{p-2} \Phi(t) d t
\end{aligned}
$$

Using the technique of Induction, we obtain:

$$
\begin{aligned}
(1+x)^{\alpha} x^{m} D_{x} I(x, \eta) & =\frac{(1+x)^{\alpha} x^{-m}}{2 \pi^{1}} \int^{b}\left\{\frac{1}{x-t+i \eta}\left[\left(\frac{t+\lambda}{x+\lambda+i \eta}\right)^{p-1}-1\right]\right. \\
& \left.-\frac{1}{x-t-i \eta}\left[\left(\frac{t+\lambda}{x+\lambda-i \eta}\right)^{p-1}-1\right]\right\}{ }_{\Phi}^{(m)}(t) d t
\end{aligned}
$$




$$
\begin{aligned}
& +\ldots \\
& +\frac{(1+x)^{\alpha} x^{m}}{2 \pi 1} \int_{a}^{b}\left[\cdot(x+\lambda+i \eta)^{-p}-(x+\lambda-i \eta)^{-p}\right](x+\lambda)^{p-2} \Phi^{(m-1)}(t) d t \\
& -\frac{p(p-1)}{2 \pi 1}(1+x)^{\alpha} x^{m} \int^{b}\left[(x+\lambda+i \eta)^{-p-1}-(x+\lambda-i \eta)^{-p-1}\right](t+\lambda)^{p-2} \Phi^{(m-2)}(t) d t \\
& +\ldots a \\
& (-1)^{m-1} \frac{p(p-1) \ldots(p+m-2)}{2 \pi^{i}}(1+x)^{\alpha} x^{m} x \\
& \int^{b}\left[(x+\lambda+i \eta)^{-p-m+1}-(x+\lambda-i \eta)^{-p-m+1}\right](t+\lambda)^{p-2} \Phi(t) d t
\end{aligned}
$$

Denote the integrals on the right-hand side of $(5.22)$ by $J_{1}, J_{2}, \ldots J_{m+1}$ in that order. In view of case $m=0, J_{1} \rightarrow 0$ as $\eta \rightarrow 0+$ uniformly for all $x \in(0, N)$. To show that other integrals converge to 0 as $\eta \rightarrow 0+$ uniformly for $x \in(0, N)$, we consider the most general integral $\mathrm{J}_{\mathrm{m}+1}$. As before,

$$
\begin{aligned}
\left|(x+\lambda+1 \eta)^{-p-m+1}-(x+\lambda-i \eta)^{-p-m+1}\right| & =\left|(-p-m+1) \int_{x+\lambda-i \eta}^{x+\lambda+1 \eta} z^{-p-m} d z\right| \\
& \leq 2 \eta|p+m-1| \frac{e^{\pi|I m p|}}{(x+\lambda)^{\operatorname{Re} p+m}}
\end{aligned}
$$

Therefore, we can find a positive constant $c$ independent of $x$ and $\eta$ such that

$$
\left|J_{m+1}\right| \leq \eta \mathrm{C} \frac{(1+x)^{\alpha_{x} m}}{(x+\lambda)^{\operatorname{Re} p+m}} \rightarrow 0 \text { as } \eta \rightarrow 0+
$$

uniformly for all $x \in(0, N)$ and each fixed $m=1,2, \ldots$

Thus, we have proved that

$$
(1+x)^{\alpha} x^{m} D^{m} I(x, \eta) \rightarrow 0 \text { as } \eta \rightarrow 0+\text { uniformly for all } x \in(0, N) \text { and each fixed }
$$
$\mathrm{m}=0,1,2,3, \ldots$

Combining this fact with inequality (4.17), we have

$$
\lim _{\eta \rightarrow 0+}\left|(1+x)^{\alpha} x^{m} D^{m} I(x, \eta)\right|<\varepsilon
$$


uniformly for all $x>0$ and each fixed $m=0,1,2, \ldots$; since $\epsilon$ is arbitrary our claim is established.

THEOREM 4.4. Let $\operatorname{Re} p>1>\alpha$ and $f(t) \in S_{\alpha}^{\prime}$. If $F(s)$ is the stieltjes transform of $f(t)$ defined by (3.1) then for $\lambda>0$ and each $\Phi \in D(I)$

$$
\lim _{\eta \rightarrow 0+}<\frac{p-1}{2 \pi i} \int_{\lambda}^{\infty}[f(y-i \eta)-F(y+i \eta)](y+t)^{p-2} d y, \Phi(t)>=0
$$

PROOF. By using the same technique as used in proving Theorem 4.1 , it can be shown that:

$$
\begin{aligned}
& <\frac{p-1}{2 \pi i} \int_{\lambda}^{\infty}[F(y-i \eta)-F(y+i \eta)](y+t)^{p-2} d y, \Phi(t)> \\
& =\int_{a}^{b}<f(x), \frac{1}{2 \pi i}\left\{\frac{1}{x-t+i \eta}\left[\left(\frac{t+\lambda}{x+\lambda+i \eta}\right)^{p-1}-1\right]\right. \\
& \left.-\frac{1}{x-t-i \eta}\left[\left(\frac{t+\lambda}{\lambda+x+i \eta}\right)^{p-1}-1\right]\right\}>\Phi(t) d t
\end{aligned}
$$

where the support of $\Phi(t)$ is contained in $(a, b), b>a>0$,

$$
\begin{aligned}
& =\left\langle f(x), \frac{1}{2 \pi i} \int_{a}^{b}\left\{\frac{1}{x-t+i \eta}\left[\left(\frac{t+\lambda}{x+\lambda+i \eta}\right)^{p-1}-1\right]\right.\right. \\
& \left.-\frac{1}{(x-t-i \eta)}\left[\left(\frac{t+\lambda}{x+\lambda-i \eta}\right)^{p-1}-1\right]\right\}>\Phi(t) d t . \quad \text { (By using Riemann's sum technique) }
\end{aligned}
$$

Letting $\eta \rightarrow 0+$, the result follows in view of Lemma 4.3.

THEOREM 4.5. For a fixed $\alpha<1<\operatorname{Re} p$, let $f(t) \in S_{\alpha}^{\prime}(I)$ and let $F(s)$ be the Stieltjes transform of $f(t)$ defined by (3.1). Then,

$$
\left.\lim _{\eta \rightarrow 0+}<\frac{\mathrm{p}-1}{2 \pi i} \int_{-\mathrm{x}}^{\lambda}(\mathrm{x}+\mathrm{t})^{\mathrm{p}-2}[\mathrm{~F}(\mathrm{t}-\mathrm{i} \eta)-\mathrm{F}(\mathrm{t}+\mathrm{i} \eta)] \mathrm{dt}, \quad \Phi(\mathrm{x})\right\rangle=\langle\mathrm{f}, \Phi\rangle
$$


for all $\Phi \in D(I)$ and $\lambda>0$.

PROOF. The result follows quite easily in view of Theorems 4.1 and 4.4.

ACKNOWLEDGEMENTS. The authors are grateful to Professor E.R. Love, for his valuable suggestions, in particular for providing the proof of Lemma 4.3. The work of the second author was supported by a National Research Council Grant Number A5298.

REFERENCES.

1. Byrne, A. and Love, E.R. Complex inversion theorems for generalized Stieltjes transforms, Australian Jr. of Math. (1975) 328-358.

2. Pandey A T., Zemanian, A.H. Complex inversion for the generalized convoluti. A 2 ansformation, Pacific Jr. of Math. 25 (1968) 147-157.

3. Pandey, J.N. On the Stieltjes transform of generalized functions, Proc. Camb. Phil. Soc. 프 (1972) 85-96.

4. Pathak, R.S. A distributional generalized Stieltjes transformation, Proceedings of Edinburgh Math. Soc. 20 (1976) 15-22.

5. Schwartz, L. Theorie des distributions, Vo1. I and II (Hermann, Paris $1957,1959)$.

6. Sumner, D.B. An inversion formula for the generalized Stieltjes transform, Bulletin American Math. Soc. 55 (1949) 174-183.

7. Tiwari, U. Some distributional transformations and Abelian theorems, Ph.D. Thesis, Carleton University (1976).

8. Widder, D.V. The Laplace transform, Princeton (1976).

9. Zemanian, A.H. Generalized integral transformation, Interscience Publishers (1968). 


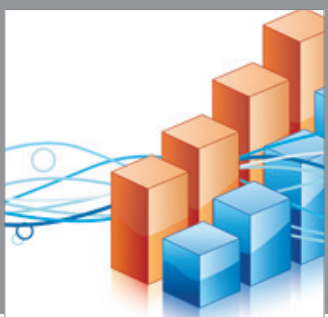

Advances in

Operations Research

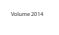

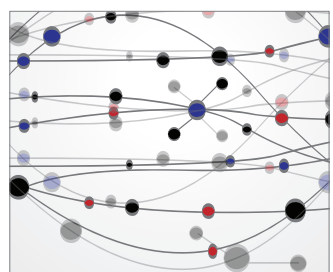

\section{The Scientific} World Journal
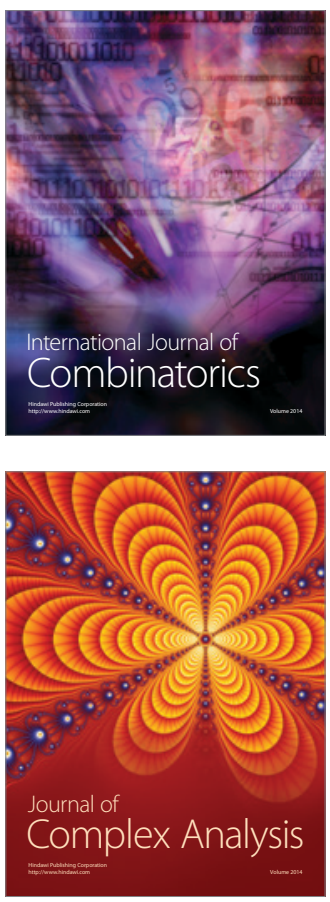

International Journal of

Mathematics and

Mathematical

Sciences
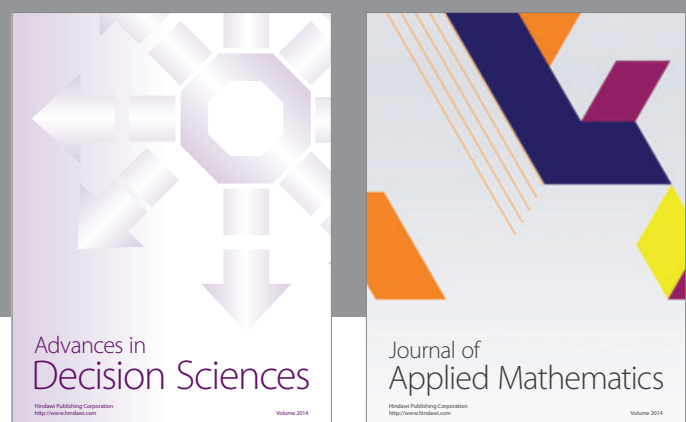

Journal of

Applied Mathematics
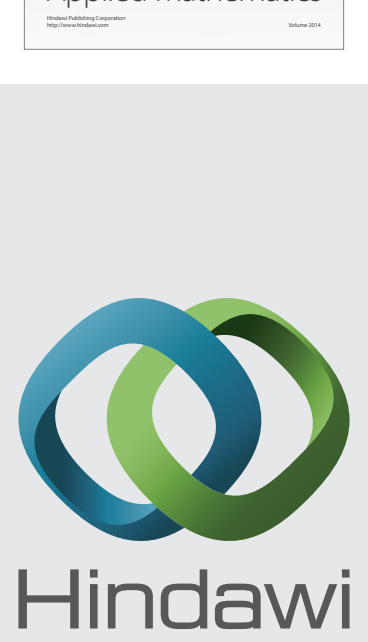

Submit your manuscripts at http://www.hindawi.com
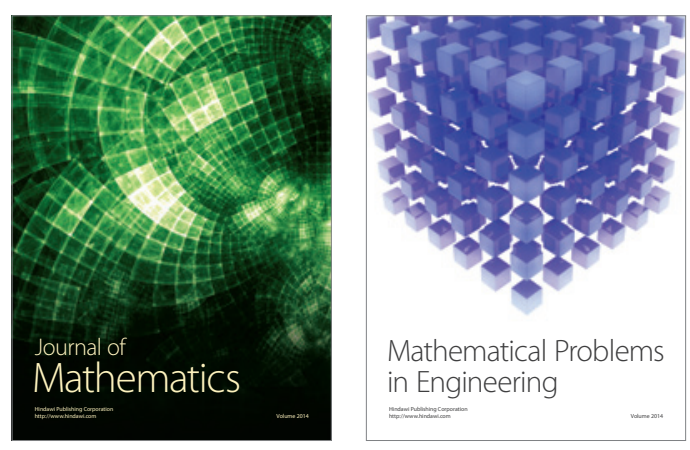

Mathematical Problems in Engineering
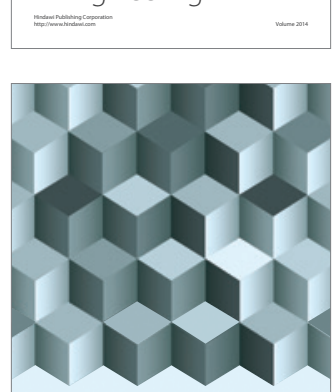

Journal of

Function Spaces
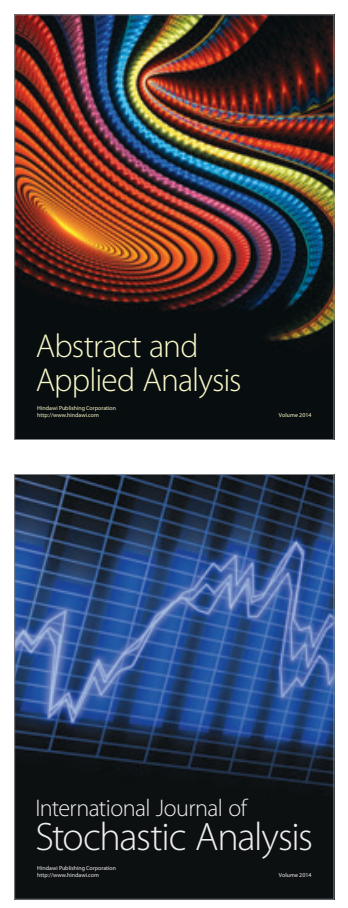

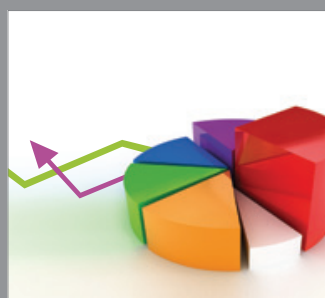

ournal of

Probability and Statistics

Promensencen
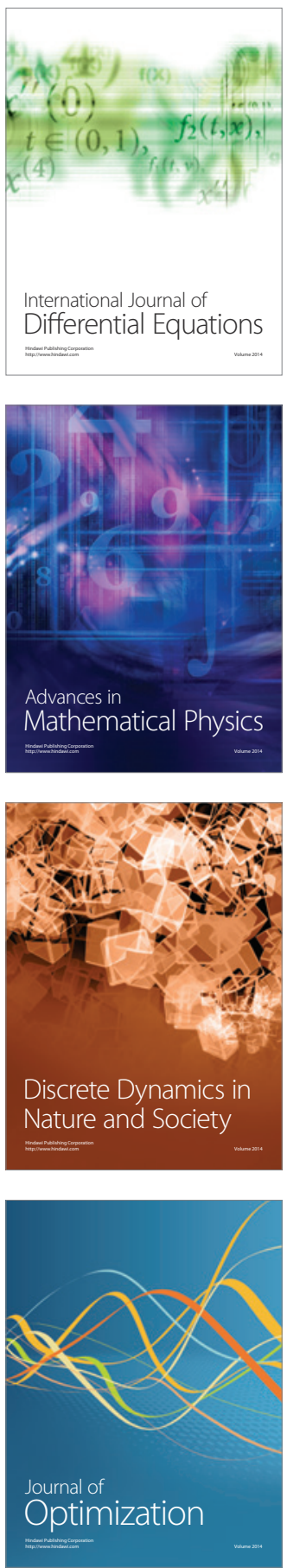